\title{
UTIUDAD DE LA HISTORIA CLINICA PARA EL DIAGNÓSTICO DE OBSTRUCCIÓN DEL TRACTO URINARIO INFERIOR EN PACIENTES CON RETENCIÓN AGUDA DE ORINA.
}

\author{
M iguel Vírseda Chamorro, Ramón Diz Rodríguez, M aria José Cancho G il, José Andrés \\ Cabrera Cabrera, Pedro Paños Lozano y Jesús Salinas Casado.
}

Servicio de Urología Hospital Central de la Defensa y Clínica Ruber Internacional. Madrid. España

\begin{abstract}
Resumen.- O BJETIVO: Determinar la utilidad diagnóstica de los datos aportados por la historia clínica para el diagnóstico de obstrucción del tracto urinario inferior (O TUI) en los pacientes con retención aguda de orina (RAO).

MÉTODO S: Se realizó un estudio transversal en una serie de 70 pacientes (19 mujeres y 51 varones) de edad media 61, 5 años (desviación típica 20,7 años), sometidos a estudio urodinámico por presentar RAO. Los pacientes fueron interrogados sobre sus a ntecedentes pa tológicos. Se les realizó además una exploración clínica, valorando el tamaño prostático en varones, presencia de prolapsos genitales en mujeres, y una exploración neurourológica. El estudio urodinámico consistió en una flujometría libre, un estudio de presión/ flujo con registro simultaneo de la actividad electromiográfica perineal, y una cistografía o videocistografía mic-
\end{abstract}

M iguel Vírseda Chamorro

C/ Argentina 4, 1요 a. 28027 M adrid. (España) e-mail: bgmeli@terra.es Trabajo recibido: 29 de septiembre 2004 ciona les. La pruebas esta dísticas utilizadas fueron el test de comparación de medias de la t de Student y el test de la Chi-cuadrado.

RESULTADO S: Los únicos datos clínicos que mostraron una relación estadísticamente significativa con la O TUI fueron: la edad, el sexo, el tamaño prostático y la presencia de lesión neurológica infrasacral (ausencia del reflejo bulbocavernoso). Los pacientes con edad superior a 74 años presentaron una sensibilidad respecto a la presencia de 0 TUI del $71 \%$ y una especificidad del $60 \%$. El sexo masculino mostró una sensibilidad del $88 \%$ y una especificidad del $36 \%$. El tamaño prostático superior o igual a un grado II, una sensibilidad del $58 \%$ y una especificidad del $82 \%$, y la ausencia de lesión infrasacral una sensibilidad del $77 \%$ y una especificidad del $48 \%$.

CONCLUSIONES: Dado que, un resultado negativo en una prueba con una alta sensibilidad, hace poco probable la presencia de la enfermedad, en el sexo femenino, la presencia de una disfunción neurógena infrasacral y una edad menor o igual a 74 años, disminuyen la probabilidad de obstrucción en pacientes con RAO. Por otra parte, como un resultado negativo para una prueba con una alta especificidad, a umenta la probabilidad de padecer la enfermedad; una prósta ta de tamaño mayoro igual a un grado II y una edad superior a los 74 años, favorecen la probabilidad de padecer obstrucción urinaria en un paciente con RAO.

Palabras clave: o bstrucción. Retención aguda de orina. Historia clínica. 
Summary.- O BJEC TIVES: To determine the diagnostic usefulness of data provided by clinical history for the diagnosis of lower urinary tract obstruction in patients with acute urinary retention.

METHODS: W e performed a transversal study in a series of 70 patients (19 women and 51 men) with a mean age of 61.5 years (typical deviation 20.7 years), who underwent urodynamic study due to acute urinary retention.

Past medical history was recorded in all patients. Physical exam was also carried out, evaluating prostate size in males, presence of genital prolapse in females, and neurourological examination. Urodynamic tests consisted on flowmetry, pressure/ flow studies with simultaneous perineal electromyography, and voiding cysto urethrogram or videocystogram. Student's $t$ mean comparison and chi-square tests were used for the statistical analysis.

RESULTS: The only clinical data that showed a statistically significant relationship with obstruction of the lower urinary tract were: age, sex, prostate size, and existence of infrasacral neurological lesion (absence of bulbocavernous reflex). Age $>74$ years showed a sensitivity of $71 \%$ for lower urinary tract obstruction and a specificity of $60 \%$. $M$ ale sex showed a sensitivity of $88 \%$ and specificity of $36 \%$. Prostate size $\geq 3 d$ grade II sensitivity was $58 \%$ and specificity $82 \%$; and absence of infrasacral lesion a sensitivity of $77 \%$ and specificity of $48 \%$.

CONCLUSION S: Provided that a negative result in a test with high sensitivity makes the likelihood of disease low, in women the presence of infrasacral neurogenic dysfunction or age $\leq 3 d 74$ years diminish the probability of obstruction in patients with acute urinary retention. $O n$ the other side, if a negative result of a test with high specificity increases the probability of having the disease, a prostate size $\geq 3 d$ grade II and age $>74$ years favour the likelihood of having urinary tract obstruction for a patient with acute urinary retention.

Keywords: 0 bstruction. Acute urinary retention. Clinical history.

\section{INTRODUCCIÓN}

La Retención Aguda de 0 rina (RA O) se define como la imposibilidad brusca para realizar el vaciado vesical. Se estima que aproximadamente una cuarta parte de los varones con hiperplasia prostática benigna padecerán este síntoma a lo largo de la evolución de su enfermedad $(1,2)$. Su incidencia anual en varones de más de 45 años estaría alrededor de 4,5/ 1000 personas-año (3), mientras que en mujeres sería de alrededor de 0,07/ 1000 personas año (4).

El tratamiento inicial de la retención aguda de orina es el drenaje urinario. Sin embargo, el tratamiento definitivo debe resolver la causa de esa retención. La retención aguda de orina no siempre se debe a una obstrucción urinaria, pues otra de las causas es la insuficiencia contráctil del detrusor (5). El estudio urodinámico es el único método diagnóstico que permite diferenciar entre ambas condiciones.

Un correcto diagnóstico de obstrucción urinaria es fundamental para el tratamiento definitivo de esta patología, pues únicamente en este caso está indicado realizar una cirugía desobstructiva, mientras que si no existe obstrucción este tipo de tratamiento está abocado al fracaso (6).

En ausencia de estudios urodinámicos, se ha valorado la información proprocionada por la historia clínica como orientativa de la presencia de obstrucción urinaria. Sin embargo, estos datos no han sido contrastados a decuadamente con un estudio urodinámico.

El objetivo de este trabajo es determinar la fiabilidad diagnóstica de los datos clínicos y demográficos respecto al diagnóstico de obstrucción en la retención aguda de orina.

\section{MATERIAL Y MÉTODOS}

Se realizó un estudio transversal de corte "cross sectional study" sobre un total de 70 pacientes que fueron remitidos para estudio urodinámico por presentar retención aguda de orina.

El estudio consistió en un interrogatorio sobre sus antecedentes patológicos, tanto médicos como quirúrgicos, la realización de una exploración clínica valorando el tamaño prostático en varones y la existencia de prolapso genital en mujeres así como una exploración neurourológica que consistió en la valoración del reflejo bulbo-cavernoso y control voluntario del esfínter anal. Se consideró que existía disfunción neurógena suprasacral si el control voluntario estaba 
alterado con conservación del reflejo bulbocavernoso. Se consideró que existía disfunción neurógena infrasacral si el reflejo bulbocavernoso estaba abolido.

El estudio urodinámico consistió en la realización de una flujometría libre seguida de un test presión detrusor/ flujo miccional, con registro simultáneo electromiográfico perineal y cistografía o videocistografía. La metodología y unidades para la realización de este estudio se ajustaron a las especificaciones propuestas por la ICS (7). El diagnóstico de obstrucción del tracto urinario en el varón se realizó de acuerdo con el nomograma provisional de presión/ flujo propuesto por la ICS (8). Para el diagnóstico de obstrucción del tracto urinario inferior femenino se empleó el nomograma propuesto por Blaivas \& Grout (9). La valoración de la contractilidad vesical en el varón se realizó de acuerdo con el nomograma propuesto por Abrams (10).

Los datos de las pacientes fueron almacenados en una base de datos Acces ${ }^{\circledR}$ y posteriormente importados informáticamente al programa estadístico SPSS versión 9 ®. Las pruebas estadísticas utilizadas fueron la prueba de la t de student de comparación de medias entre variables paramétricas y la prueba de la chi-cuadrado de comparación de proporciones. El nivel de significación se fijó en una $p=0,05$ bilateral.

\section{RESULTADOS}

\section{Estadística descriptiva}

\section{Datos demográficos}

La edad media de los pacientes de la muestra fue de 61,5 años (desviación típica de 20,7 años). La distribución por sexos fue de 19 mujeres (27\%) y 51 varones (73\%).

\section{Datos clínicos}

Los antecedentes patológicos de los pacientes y su distribución por sexos se muestran en la Tabla I. En varones fueron más frecuentes las lesiones medulares y la cirugía prostática simple. En mujeres fueron más frecuentes los antecedentes de cirugía ginecológica simple o radical.

El tacto rectal indicó la existencia de una hiperplasia benigna de próstata en 35 varones $(69 \%$ de los varones de la muestra), siendo el $40 \%$ grado I,

\begin{tabular}{|lrrrr|}
\hline \multicolumn{1}{|c}{ Antecedente* } & Mujeres & Varones & \multicolumn{1}{c|}{ Total } \\
Sin interés & & & & \\
Hiperplasia prostática & 4 & $(23 \%)$ & $13(77 \%)$ & $17(100 \%)$ \\
Traumatismo pelviano & 0 & $(0 \%)$ & $2(100 \%)$ & $2(100 \%)$ \\
Cirugía prostática simple & 0 & $(0 \%)$ & $2(100 \%)$ & $2(100 \%)$ \\
Cirugía radical intestinal & 0 & $(0 \%)$ & $6(100 \%)$ & $6(100 \%)$ \\
Cirugía radical pelviana t & 0 & $(0 \%)$ & $4(100 \%)$ & $4(100 \%)$ \\
Cirugía ginecológica simple & $3(60 \%)$ & $2(40 \%)$ & $5(100 \%)$ \\
Lesión medular congénita & $4(100 \%)$ & 0 & $(0 \%)$ & $4(100 \%)$ \\
Lesión medular adquirida & $1(100 \%)$ & 0 & $(0 \%)$ & $1(100 \%)$ \\
Ictus cerebral & $3(17 \%)$ & $15(83 \%)$ & $18(100 \%)$ \\
Ictus cerebral y prostatectomía simple & $2(33 \%)$ & $4(67 \%)$ & $6(100 \%)$ \\
Traumatismo craneoencefálico & 0 & $(0 \%)$ & $1(100 \%)$ & $1(100 \%)$ \\
Depresión & 0 & $(0 \%)$ & $1(100 \%)$ & $1(100 \%)$ \\
& $2(67 \%)$ & $1(33 \%)$ & $3(100 \%)$ \\
& Total & $19(27 \%)$ & $51(73 \%)$ & $70(100 \%)$ \\
\hline
\end{tabular}

* frecuencia absoluta (entre paréntesis porcentaje) †Histerectomía radical en mujeres o prostatectomía radical en varones. 
TABLA II. DISTRIBUCIÓ N DEL TIPO DE LESIÓN N EURO LÓ GICA POR SEXO

\begin{tabular}{|c|c|c|c|}
\hline Tipo de lesión neurológica & Mujeres & Varones & Total \\
\hline A usencia & $12(27 \%)$ & $33(73 \%)$ & $45(100 \%)$ \\
\hline Encefálica & $2(29 \%)$ & $5(71 \%)$ & $7(100 \%)$ \\
\hline M edular suprasacral & $1(14 \%)$ & $6(86 \%)$ & $7(100 \%)$ \\
\hline M edular infrasacral & $3(33 \%)$ & $6(67 \%)$ & $9(100 \%)$ \\
\hline Periférica & $1(50 \%)$ & $1(50 \%)$ & $2(100 \%)$ \\
\hline Total & $19(27 \%)$ & $51(73 \%)$ & $70(100 \%)$ \\
\hline
\end{tabular}

el $16 \%$ grado II, el $32 \%$ grado III y el $12 \%$ grado IV. La exploración vaginal apreció un prolapso vaginal en 7 mujeres (41\%), siendo de grado I en el $42 \%$, grado II en el $29 \%$ y grado III en el $29 \%$.

La exploración clínica neurourológica apreció la existencia de lesión neurológica en 25 casos (36 \% de la muestra), correspondiendo a 7 mujeres ( $41 \%$ de las mujeres de la muestra) y 18 varones (35\% de los varones de la muestra). La distribución del nivel de lesión neurológica por sexos se muestra en la Tabla II.

\section{Datos urodinámicos}

En la flujometría libre, 27 pacientes (39\% de la muestra), consiguieron realizar una micción espontánea. En el estudio presión flujo 31 pacientes(44\% de la muestra), consiguieron realizar una micción. El estudio urodinámico comprobó la presencia de obstrucción urinaria en 25 casos ( $36 \%$ de la muestra), correspondiendo en 3 casos a mujeres ( $18 \%$ del total de mujeres de la muestra) y en 16 casos a varones (31\% del total de varones de la muestra). El diagnóstico final del tipo de alteración del tracto urinario inferior se muestra en la Tabla III. El diagnóstico más común en varones fue la obstrucción prostática, y en mujeres la insuficiencia contráctil del detrusor.

\section{Estadística bivariante}

El resultado de comparar los datos demográficos y clínicos con la presencia de obstrucción se

TABLA III. DISTRIBUCIÓN DE LOS DIAG N Ó STIC O S URO DIN ÁM ICO S PO R SEXO

\begin{tabular}{|c|c|c|c|}
\hline Diagnóstico urodinámico & Mujeres & Varones & Total \\
\hline Estudio normal & $0 \quad(0 \%)$ & $3(100 \%)$ & $3(100 \%)$ \\
\hline O bstrucción prostática & $0 \quad(0 \%)$ & $7(100 \%)$ & $7(100 \%)$ \\
\hline O bstrucción por prolapso vaginal & $1(100 \%)$ & $0 \quad(0 \%)$ & $1(100 \%)$ \\
\hline O bstrucción por estenosis uretral & $1 \quad(50 \%)$ & $0 \quad(0 \%)$ & $1(100 \%)$ \\
\hline O bstrucción prostática e insuficiencia contráctil & $0 \quad(0 \%)$ & $8(100 \%)$ & $8(100 \%)$ \\
\hline O bstrucción cuello e insuficiencia contráctil & $0 \quad(0 \%)$ & $2(100 \%)$ & $2(100 \%)$ \\
\hline Hiperreflexia y disinergia cuello & $1 \quad(50 \%)$ & $1 \quad(50 \%)$ & $2(100 \%)$ \\
\hline Hiperreflexia y disinergia esfínter & $0 \quad(0 \%)$ & $4(100 \%)$ & $4(100 \%)$ \\
\hline Insuficiencia contráctil & $10 \quad(45 \%)$ & $12(55 \%)$ & $22(100 \%)$ \\
\hline Síndrome de Fowler & $1(100 \%)$ & $0 \quad(0 \%)$ & $1(100 \%)$ \\
\hline A rreflexia & $5 \quad(31 \%)$ & $11(69 \%)$ & $16(100 \%)$ \\
\hline Hiperreflexia & $0 \quad(0 \%)$ & $3(100 \%)$ & $3(100 \%)$ \\
\hline Total & $19 \quad(27 \%)$ & $51 \quad(73 \%)$ & $70(100 \%)$ \\
\hline
\end{tabular}


muestra en la tabla IV. Los únicos datos que mostraron una relación significativa con la obstrucción fueron: la edad (más edad en pacientes obstruidos), el sexo (mayor proporción de varones obstruidos), el tamaño prostático (mayor proporción de obstrucción en varones con próstatas grado II o más), y el diagnóstico clínico de disfunción neurógena infrasacral (menor porcentaje de pacientes obstruidos en los pacientes con lesión infrasacral).

El punto de corte de máxima sensibilidad y especificidad de la edad respecto al diagnóstico de obstrucción fue de 74 años (Figura 1). La sensibilidad y especificidad de cada uno de los datos clínicos significativos respecto al diagnóstico de obstrucción se muestra en la figura 2. La máxima sensibilidad correspondió al sexo masculino con un $88 \%$ (el $88 \%$ de los obstruidos fueron varones) y la máxima especificidad al tamaño prostático con un $82 \%$ (el $82 \%$ de los varones no obstruidos tenían un tamaño prostático inferior al grado II).

\section{DISCUSIÓN}

En este trabajo se observó que el $36 \%$ de los pacientes con RAO tenían obstrucción urinaria. Los únicos datos clínicos de utilidad para el diagnóstico de

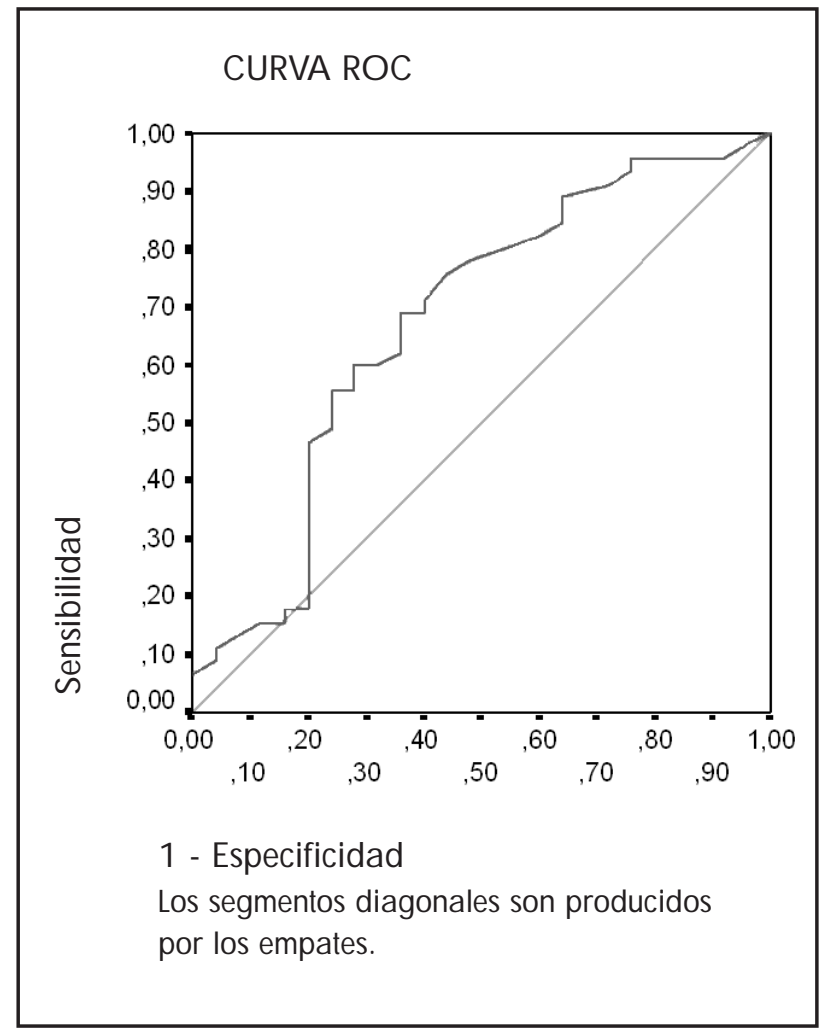

FIG URA 1. Curva de rendimiento de la edad para el diagnóstico de obstrucción del tracto urinario inferior

\section{TABLA IV. RELACIÓN DE LOS DATOS DEM O G RÁFICOS Y C ĹN IC OS CON EL DIAG N Ó STICO DE O BSTRUCCIÓ N}

\section{Datos demográficos/ clínicos}

Edad en años*

Sexo masculino $\dagger$

Próstata grado II (sólo varones) $†$

Prolapso vaginal III (sólo mujeres) †

Cirugía radical pelviana†

Cirugía prostática simple (sólo varones) †

Cirugía ginecológica simple (mujeres) $\dagger$

Lesión medular $†$

Lesión encefálica†

Depresiónt

Disfunción neurógena†

Disfunción neurógena infrasacralt

Disfunción neurógena suprasacralt

\section{Obstruidos}

$68,6(20,1)$

$22(80 \%)$

$11(58 \%)$

$1(50 \%)$

$2(22 \%)$

$3(43 \%)$

$0 \quad(0 \%)$

$7 \quad(39 \%)$

$3(37 \%)$

$1(33 \%)$

$8(32 \%)$

$5(19 \%)$

22 (33\%)
No obstruidos

$\begin{array}{rr}57,5 & (20,1) \\ 29 & (64 \%) \\ 4 & (18 \%) \\ 2 & (12 \%) \\ 7 & (79 \%) \\ 4 & (57 \%) \\ 4 & (100 \%) \\ 11 & (61 \%) \\ 5 & (62 \%) \\ 2 & (67 \%) \\ 17 & (68 \%) \\ 21 & (81 \%) \\ 44 & (67 \%)\end{array}$

\section{Significación}

$0,031 \ddagger$

$0,034 \ddagger$

$0,008 \ddagger$

0,364

0,366

0,987

0,330

0,744

0,911

0,930

0,629

$0,049 \neq$

0,572

* media (entre paréntesis desviación típica) † frecuencia absoluta (porcentaje) ‡ significativo. 
obstrucción del tracto urinario inferior en pacientes con RAO fueron el sexo, la edad, el tamaño prostático y la presencia de disfunción neurógena infrasacral.

El $88 \%$ de los pacientes obstruidos eran varones. Esto indica que la sensibilidad (porcentaje de verdaderos positivos) del dato demográfico de ser varón respecto al diagnóstico de obstrucción es alto. Sólo un $12 \%$ de los pacientes obstruidos eran mujeres (falsos positivos). Sin embargo, su especificidad (verdaderos negativos), es baja. Sólo un $36 \%$ de los pacientes no obstruidos eran mujeres, frente a un $64 \%$ de varones que tampoco estaban obstruidos (falsos negativos). Estos resultados son consecuencia de que los varones están más predispuestos a padecer RA 0 que las mujeres, como confirman otros estudios (4) (11). En nuestro estudio la proporción fue de 2,7 varones por cada mujer.

0 tros estudios utilizando muestras amplias, sitúan el porcentaje de obstrucción, en varones de más de 50 años sometidos a estudios urodinámicos, en alrededor del $62 \%$ (12). En mujeres este porcentaje estaría alrededor del 8\% (9). A plicando el teorema de Bayes a estos datos, podemos calcular que la proba-

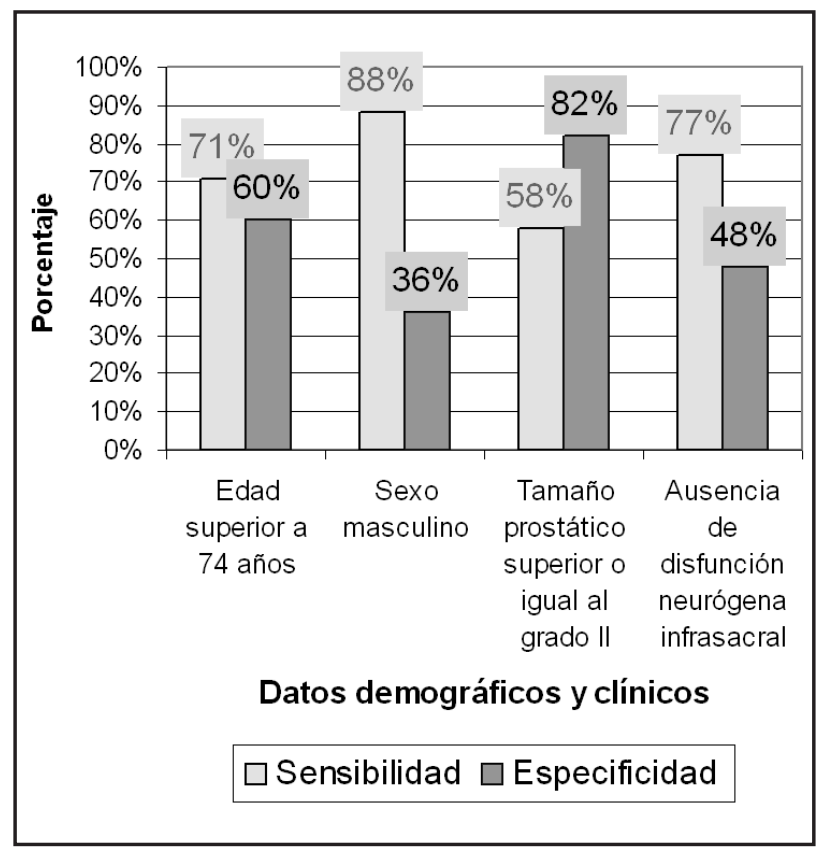

FIG URA 2. Utilidad diagnóstica de los datos demográficos y clínicos para el diagnóstico de obstrucción. bilidad de ser varón, en el caso de presentar obstrucción urinaria, en pacientes con RAO, debería ser del $94 \%$. Este porcentaje previsto es superior al porcentaje encontrado en nuestra serie. Es decir, la RA 0 incrementa la proporción de mujeres entre los pacientes obstruidos. Este aumento del porcentaje de mujeres coincide con un mayor porcentaje de pacientes obstruidas en nuestra serie (un 18\%). Este dato se puede explicar suponiendo que la obstrucción favorece la RA O. De ahí, el incremento de mujeres obstruidas.

El otro factor de riesgo demográfico fue la edad. En nuestro estudio se determinó como punto de corte una edad de 74 años. Con este valor se observó una sensibilidad del $71 \%$, siendo la especifidad algo menor (60\%). Algunos estudios han encontrado que la prevalencia de obstrucción del TUI (tracto urinario inferior), aumenta con la edad en varones (13), y en mujeres (14). El aumento del porcentaje de obstrucción con la edad en pacientes con RAO es compatible con esa relación general. Además se ha descrito un aumento del riesgo de RAO con la edad (15). Este hecho también se puede explicar suponiendo que la obstrucción favorece la RAO, ya que, al aumentar la edad también aumenta el porcentaje de pacientes obstruidos.

El tamaño prostático superior o igual a un grado II, fue un dato clínico significativamente asociado a la presencia de obstrucción en pacientes con RA O. Su sensibilidad en nuestro estudio fue baja: solo un $58 \%$ de los pacientes obstruidos tenían una próstata aumentada. Pero su especificidad fue alta: el $87 \%$ de los pacientes no obstruidos tenían una próstata menor de un grado II. El tamaño prostático también parece estar relacionado con la obstrucción del TUI en la población general (16). En series epidemiológicas también se ha demostrado una relación entre el tamaño prostático y la RAO (17), (18). Jacobsen et al (15) encuentran que los pacientes con próstatas superiores a $30 \mathrm{ml}$ tienen un riesgo relativo 3 veces mayor de presentar RAO . Estos datos confirman la hipótesis de que el aumento de riesgo de RAO en caso de incremento del tamaño prostático sea debido a la obstrucción del TUI.

La presencia de disfunción neurógena no elevó del riesgo de obstrucción en pacientes con RAO. A lgunos estudios han encontrado que el porcentaje de pacientes con obstrucción urinaria entre los que pre- 
sentan disfunción neurógena sería menor (alrededor del $19 \%$ ), siendo el $90 \%$ de las obstrucciones de naturaleza funcional (19). Aunque también, son posibles las obstrucciones prostáticas en pacientes con disfunciones neurógenas, principalmente encefálicas, como es el caso de los ictus cerebrales. En este grupo de pacientes, $\mathrm{N}$ itti et al (20) encuentran un porcentaje de obstrucciones orgánicas de alrededor del $63 \%$.

En nuestro estudio, la existencia de disfunción neurógena infrasacral disminuyó la probabilidad de presentar obstrucción urinaria. La sensibilidad de la ausencia de disfunción neurógena infrasacral respecto al diagnóstico de obstrucción fue del $77 \%$ (sólo un $23 \%$ de los pacientes obstruidos tenían esta disfunción), pero su especificidad fue baja: el $52 \%$ de los pacientes sin obstrucción tampoco tenían disfunción neurógena infrasacral. La disfunción neurógena infrasacral se asocia significativamente con la lesión de la inervación parasimpática y por tanto con la arreflexia vesical, por lo que es lógico que en estos pacientes la principal causa de RAO sea la insuficiencia contráctil del detrusor (21).

El resto de los datos clínicos examinados en este estudio no mostraron ninguna relación con el diagnóstico de obtrucción urinaria. La cirugía simple de próstata en varones puede producir alrededor de un $12 \%$ de RAO postoperatoria. Esta incidencia esta relacionada principalmente con una mala indicación de esta técnica en pacientes que no estaban previamente obstruidos (22), pero una buena indicación en pacientes obstruidos previene la aparición de RAO (18), (23). La cirugía radical pelviana sólo incrementa el riesgo de RAO si se asocia a lesión parasimpática debido al mecanismo anteriormente comentado (21).

El prolapso vaginal y la cirugía ginecológica son causas contrastadas de obstrucción urinaria en el sexo femenino, pero debido a la baja frecuencia de obstrucción urinaria en la mujer utilizando los métodos clásicos (24), este tipo de factores no parecen aumentar la probabilidad de padecer obstrucción urinaria.

\section{CONCLUSIÓN}

En conclusión, si consideramos las recomendaciones de Jaeschke et al (25), según las cuales, cuando una prueba presenta una sensibilidad muy alta, un resultado negativo para esa prueba hace poco probable la presencia de la enfermedad: en el sexo femenino, la presencia de una disfunción neurógena infrasacral y una edad menor de 74 años disminuyen la probabilidad de obstrucción en pacientes con RAO. Por otra parte, si cuando una prueba presenta una especificidad muy alta, un resultado negativo para esa prueba aumenta la probabilidad de padecer la enfermedad, se deduce que: una próstata de tamaño mayor o igual a un grado II y una edad superior a los 74 años aumentan la probabilidad de padecer obstrucción urinaria en un paciente con RAO .

\section{BIBUOGRAFIA y LECTURAS RECOMENDADAS (*lectura de interés y $* *$ lectura fundamental)}

1. SERRETA, V.; MORGIA, G.; FONDACARO, L. y cols: "Members of the Sicilian-Calabrian Society of Urology. Management of symptomatic benign prostatic hyperplasia in southern Italy: a retrospective analysis of the Sicilian-Calabrian Society of Urology (SSCU) of 32,000 patients". Urol. Int., 71: 16, 2003.

2. ROEHRBORN, C.G.: "Reporting of acute urinary retention in $\mathrm{BPH}$ treatment trials: importance of patient follow-up after discontinuation and case definitions". Urology, 59: 811, 2002.

3. MEIGS, J.B.; BARRY, M.J.; GIOVANNUCCI, E. y cols.: "Incidence rates and risk factors for acute urinary retention: the health professionals followup study". J. Urol., 162: 376, 1999.

4. VAN DER LINDEN, E.F.; VENEMA, P.L.: "Acute urinary retention in women". Ned. Tijdschr. Geneeskd., 142: 1603, 1998.

5. CURTIS, L.A.; DOLAN, T.S.; CESPEDES, R.D.: "Acute urinary retention and urinary incontinence". Emerg. Med. Clin. North Am., 19: 591, 2001.

6. DJAVAN, B.; MADERSBACHER, S.; KLINGLER, C. y cols.: "Urodynamic assesment of patients with acute urinary retention: is treatment failure after prostatectomy predictable?". J. Urol., 158: 1829, 1997.

7. SCHÄFER, W.; ABRAMS, P.; LIA, L. y cols.: "Good urodynamics practices: uroflowmetry, filling cystometry, and pressure-flow studies". Neurourol Urodyn, 21: 261, 2002.

8. GRIFFIHTS, D.; HÖFNER, K.; VAN MASTRIGT, R. y cols.: "Standardisation of terminology of lower urinary tract function: pressure-flow studies of voiding, urethral resistance and urethral obstruction". Neurourol Urodyn, 16: 1, 1997.

*9. BLAIVAS, J.; GROUTZ, A.: "Bladder outlet obstruction nomogram for women with lower urinary tract symptomatology". Neurourol Urodyn,19: 553, 2000.

*10. ABRAMS, P.: "Bladder otulet obstruction index, bladder contractility index and bladder voiding efficiency:three simple indices to define bladder voiding function”. BJU.,84: 14, 1999.

11. MEIGS, J.B.; BARRY, M.J.; GIOVANNUCCI, E. y cols.: "Incidence rates and risk factors for acute urinary retention: the health professionals followup study”. J. Urol., 162: 376, 1999. 
12. KAPLAN, S.A.; BOWERS, D.L.; TE, A.E. y cols.: "Differential diagnosis of prostatism: A 12-year retrospective analysis of symptoms, urodynamics and satisfaction with therapy". J. Urol., 155: 1305, 1996.

13. KNUTSON, T.; EDLUND, C.; FALL, M. y cols.: "BPH with coexisting overactive bladder dysfunction-an everyday urological dilemma". Neurourol Urodyn., 20: 237, 2001.

14. YANG, J.M.; HUANG, W.C.: "Factors associated with voiding dysfunction in women with lower urinary tract symptoms: A mathematical model explanation". Neurourol Urodyn., 22: 574, 2003.

15. JACOBSEN, S.J.; JACOBSON, D.J.; GIRMAN, C.J. y cols.: "Natural history of prostatism: risk factors for acute urinary retention". J. Urol., 158: 481, 1997.

16. MADERSBACHER, S.; KLINGLER, H.C.; DJAVAN, B. y cols.: "Is obstruction predictable by clinical evaluation in patients with lower urinary tract symptoms?". Br. J. Urol., 80: 72, 1997.

17. ROEHRBORN, C.G.; MALICE, M.; COOK, T.J. y cols.: "Clinical predictors of spontaneous acute urinary retention in men with LUTS and clinical BPH: a comprehensive analysis of the pooled placebo groups of several large clinical trials". Urology, 58: 210, 2001.

18. KIRBY, R.S.: "The natural history of benign prostatic hyperplasia: what have we learned in the last decade?". Urology, 56: 3, 2000.

19. SAKAKIBARA, R.; HATTORI, T.; UCHIYAMA, T. y cols.: "Neurogenic failures of the external urethral sphincter closure and relaxation; a videourodynamic study". Auton. Neurosci. 86: 208, 2001.

20. NITTI, V.W.; ADLER, H.; COMBS, A.J.: "The role of urodynamics in the evaluation of voiding dysfunction in men after cerebrovascular accident". J. Urol., 155: 263, 1996.

*21. ESTEBAN FUERTES, M.; SALINAS CASADO, J.; RESEL ESTEVEZ, L. Y cols.: "Disfunción neurógena vesicouretral postquirúrgica. Aspectos clínicos y conceptuales basados en el análisis de una serie de 152 pacientes". Arch. Esp. Urol., 51: 901, 1998.

22. REYNARD, J.M.; SHEARER, R.J.: "Failure to void after transurethral resection of the prostate and mode of presentation". Urology, 53: 336, 1999.

*23. DUBEY, D.; KUMAR, A.; KAPOOR, R. y cols.: "Acute urinary retention: defining the need and timing for pressure-flow studies". BJU Int., 88: 178, 2001.

24. GRIFFITHS, D.J.: "Pressure-flow studies of micturition". Urol. Clin. North Am., 23: 279, 1996.

**25. JAESCHKE, R.; GUYANT, G.H.; SACKETT, D.I.: "How to use an article about diagnostic test. What are the results and will help me in caring for my patients?" JAMA, 271:703, 1994. 